\title{
Propranolol Hydrochloride
}

National Cancer Institute

\section{Source}

National Cancer Institute. Propranolol Hydrochloride. NCI Thesaurus. Code C29382.

The hydrochloride form of propranolol, a synthetic beta-adrenergic receptor blocker with antiang inal, antiarrhythmic, and antihypertensive properties. Propranolol competitively antagonizes beta-adrenergic receptors, thereby inhibiting beta-adrenergic reactions, such as vasodilation, and negative chronotropic and inotropic effects. 